Searching for prompt signatures of nearby core-collapse supernovae by a joint analysis of neutrino and gravitational wave data

This article has been downloaded from IOPscience. Please scroll down to see the full text article.

2010 Class. Quantum Grav. 27084019

(http://iopscience.iop.org/0264-9381/27/8/084019)

View the table of contents for this issue, or go to the journal homepage for more

Download details:

IP Address: 131.215.220.185

The article was downloaded on 21/10/2010 at 16:29

Please note that terms and conditions apply. 


\title{
Searching for prompt signatures of nearby core-collapse supernovae by a joint analysis of neutrino and gravitational wave data
}

\author{
I Leonor $^{1}$, L Cadonati ${ }^{2}$, E Coccia $^{3,4}$, S D'Antonio ${ }^{4}$, A Di Credico ${ }^{3}$, \\ V Fafone $^{4}$, R Frey ${ }^{1}$, W Fulgione ${ }^{5}$, E Katsavounidis ${ }^{6}$, C D Ott ${ }^{7}$,

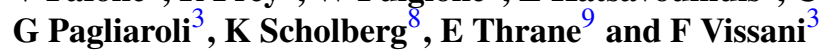 \\ ${ }^{1}$ University of Oregon, Eugene, OR 97403, USA \\ 2 University of Massachusetts, Amherst, MA 01003, USA \\ 3 INFN Laboratori Nazionali del Gran Sasso, Assergi, Italy \\ ${ }^{4}$ Università di Roma Tor Vergata, Roma, Italy \\ 5 INAF, Torino, Italy \\ ${ }^{6}$ Massachusetts Institute of Technology, Cambridge, MA 02139, USA \\ ${ }^{7}$ California Institute of Technology, Pasadena, CA 91125, USA \\ ${ }^{8}$ Duke University, Durham, NC 27708, USA \\ ${ }^{9}$ University of Minnessota, Minneapolis, MN 55455, USA \\ E-mail: ileonor@uoregon.edu
}

Received 14 November 2009, in final form 30 December 2009

Published 6 April 2010

Online at stacks.iop.org/CQG/27/084019

\begin{abstract}
We discuss the science motivations and prospects for a joint analysis of gravitational wave $(\mathrm{GW})$ and low-energy neutrino data to search for prompt signals from nearby supernovae ( $\mathrm{SNe}$ ). Both gravitational wave and low-energy neutrinos are expected to be produced in the innermost region of a core-collapse supernova, and a search for coincident signals would probe the processes which power a supernova explosion. It is estimated that the current generation of neutrino and gravitational wave detectors would be sensitive to galactic corecollapse supernovae, and would also be able to detect electromagnetically dark SNe. A joint GW-neutrino search would enable improvements to searches by way of lower detection thresholds, larger distance range, better live-time coverage by a network of GW and neutrino detectors, and increased significance of candidate detections. A close collaboration between the $\mathrm{GW}$ and neutrino communities for such a search will thus go far toward realizing a much soughtafter astrophysics goal of detecting the next nearby supernova.
\end{abstract}

PACS numbers: $04.30 . \mathrm{Tv}, 04.80 . \mathrm{Nn}$, 95.30.Cq, 95.30.Sf, 95.55.Vj, 95.55.Ym, 95.85.Ry, 95.85.Sz, 97.60.Bw 


\section{Motivation}

The predicted rate of core-collapse supernovae $(\mathrm{SNe})$ in our galaxy is $\sim 2$ per century; the rate is about twice the galactic rate out to the Andromeda galaxy $(\sim 1 \mathrm{Mpc})$, and about one per year out to the Virgo cluster $(\sim 10 \mathrm{Mpc})[1]$. These numbers are accompanied by large uncertainties inherent in converting galaxy properties to supernova rates. However, in [1] it is also suggested that the increased number of nearby core-collapse supernovae discovered within the past few years strongly indicates that the predicted rates might be significantly underestimated by a factor of $\sim 3$ in the $3-5 \mathrm{Mpc}$ range. A direct upper limit on the galactic $\mathrm{SNe}$ rate, based on non-observations of antineutrino events in the past 25 years, is given in [2].

Contemporary neutrino detectors and kilometer-scale gravitational wave (GW) detectors are currently poised to detect the next galactic core-collapse supernova. Both neutrino and gravitational wave signals are expected to be generated in the innermost region of a dying star, and both signals are expected to be emitted within a short time interval of each other, i.e. within a few milliseconds $[3,4]$. While both neutrino and GW detectors are preparing to independently detect a galactic supernova, there are science benefits to systematically searching for a supernova signature using a joint analysis of neutrino and GW data which is guided by the expected proximity of the neutrino and GW signals. These science benefits include lower detection threshold requirements, better live-time coverage, increased significance of candidate detections, extended distance reach to the local volume of galaxies and increased sensitivity to core-collapse events which have only a weak or non-existent electromagnetic signature.

The detection of a burst of low-energy neutrinos from SN1987A, at a distance of about $50 \mathrm{kpc}$ in the large magellanic cloud, by the Kamiokande II and Irvine-Michigan-Brookhaven (IMB) experiments, and by scintillation neutrino detectors [5-9] demonstrated the capability of neutrino detectors to detect SN events, and paved the way for a description and validation of the standard model of neutrino emission from a core-collapse supernova. In a core-collapse supernova, $\sim 99 \%$ of the neutron star's gravitational binding energy $\left(\sim 3 \times 10^{53} \mathrm{ergs}\right)$ is released in the form of neutrinos (and antineutrinos) of all flavors. These neutrinos have energies in the few tens of $\mathrm{MeV}$ and are emitted over a time scale of a few tens of seconds. The neutrino light curve is expected to show structure, with an increase in luminosity during the first $\sim 0.5 \mathrm{~s}$ due to accretion of matter onto the proto-neutron star [10-12]. A neutronization burst, which is a peak in the $v_{e}$ luminosity produced as the shock from the core bounce propagates through the star's outer core, is expected to last a few milliseconds after the core bounce. However, the energy emitted in the neutronization burst is only $\lesssim 1 \%$ of the total energy emitted, and such a feature might not be easily recognized in a measured neutrino light curve because of its short duration, and because the cross section of $v_{e} e$ scattering is lower than that of the dominant inverse beta decay $\left(\bar{v}_{e} p \rightarrow e^{+} n\right)$ reaction in a neutrino detector's medium [10]. The events detected by Kamiokande II and IMB from SN1987A are reproduced in figure 1.

The groundbreaking discovery of a neutrino burst from SN1987A was guided by an optical sighting of the supernova [5, 7]. The optical sighting of such a close astrophysical event motivated the analysis of archived neutrino data and guided the time scale in which to perform the search. It is plausible, however, that a fraction of core-collapse events are accompanied only by a faint electromagnetic display. This might be due to extinction brought about by an extremely dusty environment or the intervening interstellar medium, or an inherently weak accompanying electromagnetic emission with a fast decay time [13]. For example, the supernova of Cassiopeia A, one of the youngest known galactic supernova remnants and which is at a relatively close distance of $3.4 \mathrm{kpc}$, has no historical record of widespread sighting $[14,15]$ during the epoch when the explosive fireworks would have reached Earth 


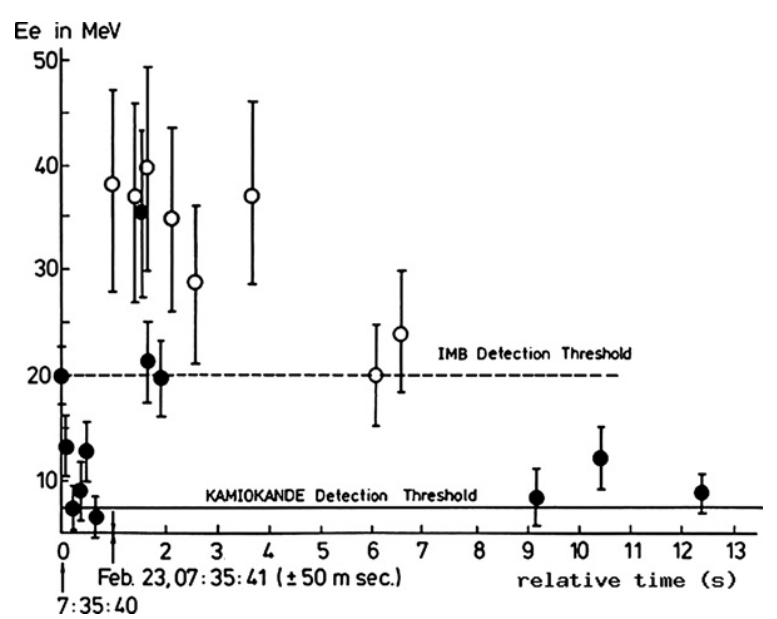

Figure 1. The energy of the events detected by Kamiokande II (closed circles) and IMB (open circles), produced by the neutrino burst from SN1987A, as a function of time, in seconds [7].

around 340 years ago, and it is plausible that this supernova fell in this category [14, 16, 17]. Obscuration by dust could also explain the non-sighting of the supernova of an even younger, $\lesssim 150$ year-old remnant, G1.9+0.3 [18]. This would be analogous to how the dearth of known galactic supernova remnants could possibly be attributed to low surface brightness, leaving faint supernova remnants unresolved from the galactic background emission [19].

It has also been argued that observations suggest a deficit of optically observed high-mass ( $25 \mathrm{M}_{\odot}$ ) core-collapse SN progenitors [13], and that optical searches have provided little information on the possibility that massive stars end their lives by forming black holes without the dramatic electromagnetic signature of an explosion. In [13], it is estimated that an upper bound on the rate of the so-called 'failed' supernovae is roughly equal to the rate of successful $\mathrm{SNe}$, and that the lower bound on black hole formation rate is $\sim 25 \%$ that of normal SNe.

A joint GW-neutrino search for nearby core-collapse supernovae could potentially provide insight to this scenario where a considerable fraction of stars end their lives with little or no electromagnetic display.

Indeed, the natural progression of the expected supernova signature-from the prompt GW and neutrino signals to the optical signal which is expected to rise many minutes to hours later-and the current state of neutrino and gravitational wave detectors and their respective detection algorithms, make it likely that the detection of the next nearby supernova will proceed in a direction opposite that of SN1987A, i.e. that prompt neutrino and GW detections would trigger optical telescopes to search for an optical counterpart. The infrastructure of the Supernova Early Warning System (SNEWS), for example, is designed to alert observatories in the event of a detection of neutrinos from a nearby supernova [20, 21]. Simulations also indicate that, for a galactic supernova, the neutrino detectors Super-Kamiokande and IceCube would be able to reconstruct the SN bounce time to within a few milliseconds [22, 23]. On the gravitational wave side, there is also an alert system, called LOOC UP [24], which is being developed to send plausible future candidate $\mathrm{GW}$ triggers to optical observatories for confirmation of a corresponding astrophysical source.

Astrophysical events-such as gamma-ray bursts (GRBs) and flares from soft gamma repeaters (SGRs) — detected by other observatories have been extensively utilized as external triggers in the analysis of LIGO-Virgo data to search for GW counterparts to these events 
[25-30]. The strategy of using external triggers with precise event timing and position information to look for GW signals is motivated mainly by the decrease in both background rate and the effective number of experimental trials that shorter analysis time windows make possible. In the case of GRBs and SGRs, the analysis windows range from a few seconds to a few minutes. On the other hand, the use of an optical signal from a supernova as an external trigger does not provide the same tight constraints on the time and duration of the analysis window. Studies indicate that, at best, the time of a supernova explosion can be determined from an optical light curve to within a few hours, but only if the first measurement of the optical flux is made within a day of the explosion [31].

In contrast, the gravitational wave and neutrino signals are expected to be detected within a tight window, ranging from a few milliseconds to a few seconds, depending on the dominant GW emission process [3, 4].

\section{Science benefits of a joint GW-neutrino search}

Several of the world's neutrino detectors have performed searches for core-collapse supernovae and have evaluated their respective detection efficiencies as a function of distance. The SuperKamiokande (Super-K) water Cherenkov detector in Japan [32], the scintillation detectors Large Volume Detector (LVD) [33] and Borexino [34] in Italy, and KamLAND in Japan [35], IceCube at the South Pole [36], MiniBooNE in the USA [37], and others [38] are expected to robustly detect a neutrino burst from a galactic supernova in the baseline model scenario. Super-K, for example, would detect $\sim 8000$ events for a core-collapse SN at the center of the Milky Way, $\sim 8.5 \mathrm{kpc}$ away [32]. The Baksan scintillation detector had previously also performed a systematic search [39].

Analogously, all-sky searches for GW bursts have been performed using data from the Laser Interferometer Gravitational Wave Observatory (LIGO), the most recent of which made use of data from the fourth (S4) and fifth (S5) LIGO science runs [40, 41]. While no gravitational waves have been directly detected from an astrophysical source, the current generation of LIGO-Virgo interferometric GW detectors have made tremendous progress in improving their sensitivities [42-46], and are expected to be sensitive to several models of GW emission from a galactic core-collapse supernova [3, 4]. Significant improvements in sensitivity are expected to continue with the anticipated advent of the next generation of GW interferometers, Advancd LIGO and Virgo. However, while the astrophysical motivation for expecting gravitational waves to accompany core-collapse supernovae is strong, the expected rate, gravitational wave strength and waveform morphology are uncertain [3, 4]. As a benchmark, the expected energy going into gravitational waves may range from $10^{-10}$ to $10^{-4} \mathrm{M}_{\odot} c^{2}$ (or $2 \times 10^{44}$ to $2 \times 10^{50} \mathrm{ergs}$ ), and thus only a small fraction of the energy liberated in neutrinos.

Estimating the sensitivity improvement of a gravitational wave search due to a tighter search window and lower background rate requires assumptions on the spectrum of the background events. In the all-sky GW burst search using the first-year data of LIGO's fifth science run, a false alarm rate of 1 in 100 years in the $64-200 \mathrm{~Hz}$ frequency band corresponds to a three-detector network signal-to-noise ratio $(\mathrm{SNR})$ threshold of $\approx 8.5$. For frequencies above $200 \mathrm{~Hz}$, the corresponding SNR threshold is lower, but the interferometers' strain sensitivity is lower at this frequency band (cf figure 5 and figure 2 of [41], and also appendix E of [41]). Requiring a coincidence of GW events within $\mathrm{O}(1 \mathrm{~s})$ of a neutrino signal tuned at a rate of 1 per day would allow GW detectors to operate at a false alarm rate of $3 \times 10^{-5} \mathrm{~Hz}$, which in turn corresponds to a SNR threshold of $\approx 3.5$ [41], or an improvement of a factor of $\sim 2$ in sensitivity. The distance reach of gravitational wave detectors scales linearly with the inverse 


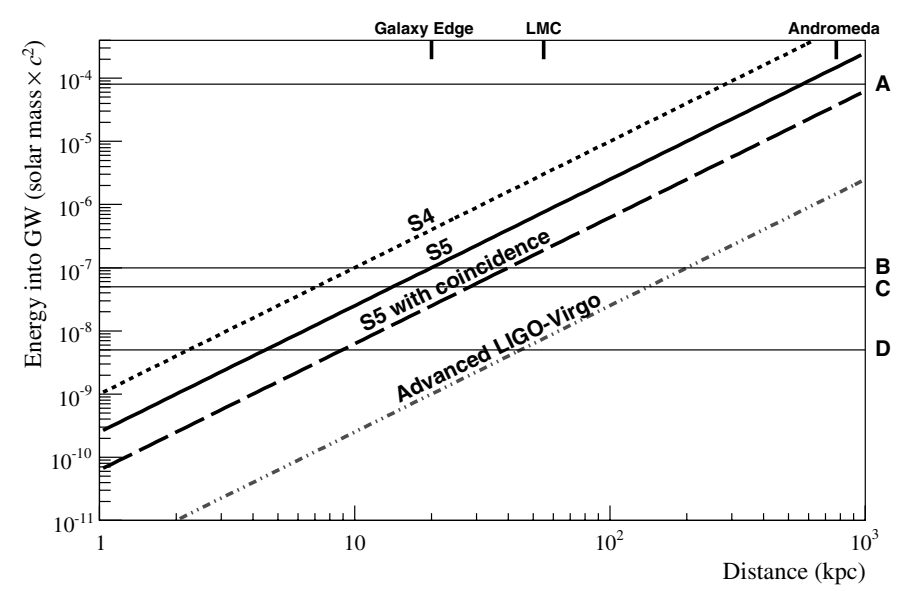

Figure 2. Sensitivity of gravitational wave detectors to core-collapse supernovae as limited by the efficiency of past, present and foreseen searches. The diagonal lines in this log-log plot reflect the fundamental relation connecting energy going into gravitational waves, distance to the source and root-sum-square strain sensitivity of a search, $h_{\mathrm{rss}}$ (cf equation (1)). These lines correspond to a fixed search sensitivity at the gravitational wave detectors for narrow-band signals in the most sensitive frequency region $(\sim 150 \mathrm{~Hz})$. All combinations of energy-distance above and to the left of these diagonal lines can be probed in a search. The dotted line identified as ' $\mathrm{S} 4$ ' corresponds to LIGO's fourth science run, and the solid line labeled 'S5' to LIGO's fifth science run. The dashed line reflects the estimated improvement in sensitivity in a joint GW-neutrino search. The dot-dashed line is the expected reach of Advanced LIGO and Virgo, with no assumption of joint searches with neutrinos [43]. The horizontal lines represent upper bounds on the energy release for four core-collapse models (as summarized in [4]): A: PNS pulsations (acoustic mechanism, [47]); B: rotational instability; C: rotating collapse and bounce; D: convection and SASI.

of SNR. Such potential improvement in gravitational wave sensitivity, in a joint GW-neutrino search, will increase the science reach of the GW instruments relative to what they can achieve alone.

To quantify the science reach, it is important to appreciate that the guidance from source phenomenology is subject to significant uncertainties [4]. All estimates of GW bursts associated with supernovae rely on models (see [47-53] for recent GW emission estimates). Most such models are not yet three-dimensional, do not incorporate the entire set of possibly relevant physics and, most importantly, do not (in most cases) predict robust supernova explosions as observed in the electromagnetic universe. Moreover, none of the current 'stateof-the-art' simulations can make reliable predictions of the mechanism responsible for the observed velocities of pulsars of up to $1000 \mathrm{~km} \mathrm{~s}^{-1}$ (but see [54] who do predict such kicks, albeit with a simplified model). It is likely that these velocities were imparted on the neutron stars at birth ('pulsar birth kicks') which obviously must involve multi-dimensional dynamics and gravitational wave emission presently not accounted for in models. Thus, despite the availability of multiple potential explosion mechanisms and their associated multidimensional dynamics and gravitational wave signatures, the current picture is unlikely to be complete. Moreover, the current, most pessimistic estimates are probably overly so in predicting the gravitational wave yield of core-collapse supernovae. In the absence of complete models, observations can and must guide our understanding of the astrophysical systems. Joint analysis of neutrino data with initial and enhanced LIGO-Virgo observations 
would significantly enhance the capability to constrain models of gravitational wave emission in core-collapse events.

For isotropic emission of gravitational waves, the luminosity distance is related to the energy emitted by the source in $\mathrm{GW}$ waves, $E_{\mathrm{GW}}$, and to the root-sum-square strain amplitude at the detector, $h_{\mathrm{rss}}$, by [40],

$$
E_{\mathrm{GW}} \approx \frac{\pi^{2} c^{3}}{G} D^{2} f_{o}^{2} h_{\mathrm{rss}}^{2},
$$

where $f_{o}$ is the observed frequency of the waves. For a hypothetical source at a galactic distance of $10 \mathrm{kpc}$ and an assumed signal morphology of a sine-Gaussian waveform [41] with central frequency $153 \mathrm{~Hz}$ and quality factor $Q=9$ (where $Q$ is a dimensionless quantity which is roughly a measure of the width of the waveform in terms of number of cycles of the sinusoid), the mass sensitivity during the LIGO S5 run is $1.9 \times 10^{-8} \mathrm{M}_{\odot} c^{2}$ [41]. The proposed joint search, with a factor of $\sim 2$ improvement in sensitivity, would decrease by a factor of $\sim 4$ the minimum energy which is probed in core-collapse supernovae, i.e. down to $4 \times 10^{-9} \mathrm{M}_{\odot} c^{2}$ for a galactic supernova with signal content in the most sensitive band of the gravitational wave detectors. Searches at higher frequencies would be penalized by the $f_{o}^{2}$ dependence and by lower strain sensitivity of the detectors at these frequencies. At the same time, however, such searches are characterized by a lower accidental background rate [41], and the improvement in sensitivity which could be achieved at higher frequencies would be about the same as that which was estimated for searches at the instruments' most sensitive frequency, i.e. a factor $\sim 2$ improvement (cf figure 5 of [41]). These scaling laws, and the potential improvement in science reach offered by a joint search, are summarized in figure 2, together with the expected gravitational wave emission in four sample emision mechanisms.

A gravitational wave coincidence requirement also has the potential to improve the sensitivity of neutrino experiments by relaxing the criteria for detection. For example, SuperK's recent 'distant' burst search [32] requires two neutrino events (with energy threshold $17 \mathrm{MeV}$ ) within $20 \mathrm{~s}$, which corresponds to approximately $8 \%$ probability of detecting a supernova in Andromeda. The accidental coincidence rate for this criterion is less than one per year; the single event rate at this threshold is about 1 per day. If one could achieve an acceptable accidental rate by requiring coincidence of a single neutrino event with a gravitational wave signal, then the probability of a core-collapse event in Andromeda satisfying the search criterion would be about $35 \%$, as shown in figure 3. Distant burst search parameters could be re-optimized with respect to current ones; the neutrino event energy threshold could potentially be reduced, further improving sensitivity.

\section{Summary}

We have motivated the search for nearby core-collapse supernovae using a joint analysis of low-energy neutrino and gravitational wave data, and we have shown examples of the science benefits of such a joint analysis. Turning this idea into a reality in the immediate future using contemporary neutrino and gravitational wave data would make possible a richer exploration of the innermost, dynamical processes in a core-collapse supernova. A search like this is a necessary complement to the joint high-energy neutrino and gravitational wave search that is currently being planned [55]. Moreover, embarking on such a task now would be forward looking, since this kind of analysis would gain importance as the sensitivities of experiments improve. The Advanced LIGO and Virgo detectors [56] are expected to start operating in 2014, and are designed to improve the sensitivity of the initial detector configurations by a factor of $\sim 10$. At the same time, there are a number of large future neutrino experiments 


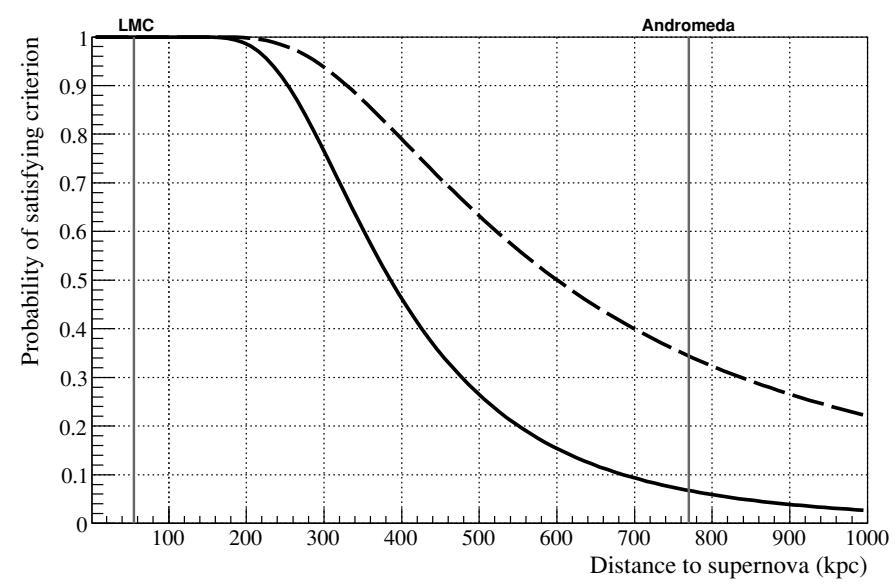

Figure 3. Estimated probability of satisfying a Super-K neutrino burst search criterion as a function of distance. Solid curve: standard search parameters [32]. Dashed curve: probability if only a single neutrino event is required, in coincidence with a GW signal.

planned, employing various technologies, including some of megaton scale [57-61]. In [1], it is pointed out that such detectors will be able to observe on the order of one supernova neutrino event every few years from beyond the local group of galaxies ( $2 \mathrm{Mpc})$. In such a regime, some kind of external (non-neutrino) trigger will be essential to distinguish supernova neutrino-induced events from background.

\section{Acknowledgments}

CD Ott is partly supported by the National Science Foundation under grant number AST0855535 .

\section{References}

[1] Ando S, Beacom J F and Yüksel H 2005 Phys. Rev. Lett. 95171101

[2] Strumia A and Vissani F 2007 arXiv:hep-ph/0606054v2

[3] Ott C D 2009 Class. Quantum Grav. 26204015

[4] Ott C D 2009 Class. Quantum Grav. 26063001

[5] Hirata K et al 1987 Phys. Rev. Lett. 581490

[6] Bionta R M et al 1987 Phys. Rev. Lett. 581494

[7] Koshiba M et al 1988 Supernova 1987A in the Large Magellanic Cloud (Cambridge: Cambridge University Press) p 130

[8] Alekseev E N, Alekseeva L N, Volchenko V I and Krivosheina I V 1987 J. Exp. Theor. Phys. Lett. 45589

[9] Aglietta M et al 1987 Europhys. Lett. 31315

[10] Totani T, Sato K, Dalhed H E and Wilson J R 1998 Astrophys. J. 496216

[11] Loredo T J and Lamb D Q 2002 Phys. Rev. D 65063002

[12] Pagliaroli G, Vissani F, Constantini M L and Ianni A 2009 Astropart. Phys. 31163

[13] Kochanek C S, Beacom J F, Kistler M D, Prieto J L, Stanek K Z, Thompson T A and Yüksel H 2008 Astrophys. J. $\mathbf{6 8 4} 1336$

[14] da Silva L A L 1993 Astrophys. Space Sci. 202215

[15] Hughes D W 1980 Nature 285132

[16] Krause O, Birkmann S M, Usuda T, Hattori T, Goto M, Rieke G H and Misselt K A 2008 Science 3201195

[17] Young P A, Fryer C L, Hungerford A, Arnett D, Rockefeller G, Timmes F X, Voit B, Meakin C and Eriksen K A 2006 Astrophys. J. $\mathbf{6 4 0} 891$ 
[18] Green D A, Reynolds S P, Borkowski K J, Hwang U, Harrus I and Petre R 2008 Mon. Not. R. Astron. Soc. 387 L54

[19] Green D A 2005 Mem. Soc. Astron. Ital. 76534

[20] Antonioli P et al 2004 New J. Phys. 6114

[21] Scholberg K 2008 Astron. Nachr. 329337

[22] Pagliaroli G, Vissani F, Coccia E and Fulgione E 2009 Phys. Rev. Lett. 103031102

[23] Halzen F and Raffelt G G 2009 Phys. Rev. D 80087301

[24] Kanner J, Huard T L, Márka S, Murphy D C, Piscionere J, Reed M and Shawhan P 2008 Class. Quantum Grav. 25184034

[25] Abbott B et al 2009 arXiv:0908.3824

[26] Abbott B et al 2008 Astrophys. J. 6811419

[27] Abbott B et al 2008 Phys. Rev. D 77062004

[28] Abbott B et al 2005 Phys. Rev. D 72042002

[29] Abbott B et al 2009 Astrophys. J. 701 L68

[30] Abbott B et al 2008 Phys. Rev. Lett. 101211102

[31] Cowen D F, Franckowiak A and Kowalski M 2009 arXiv:0901.4877v1

[32] Ikeda M et al 2007 Astrophys. J. 669519

[33] Agafonava N Y et al 2008 Astropart. Phys. 28516

[34] Cadonati L, Calaprice F P and Chen M C 2002 Astropart. Phys. 16361

[35] Piepke A et al 2001 Nucl. Phys. B 9199

[36] Kowarik T et al 2009 Proc. of the 31st ICRC (arXiv:0908.0441)

[37] Aguilar-Arevalo A A et al 2009 arXiv:0910.3182

[38] Scholberg K 2008 arXiv:astro-ph/0701081

[39] Alexeyev E N and Alexeyeva L N 2002 J. Exp. Theor. Phys. 955

[40] Abbott B et al 2007 Class. Quantum Grav. 245343

[41] Abbott B et al 2009 Phys. Rev. D 80102001

[42] Abbott B P et al 2009 Rep. Prog. Phys. 72076901

[43] Smith J R et al 2009 Class. Quantum Grav. 26114013

[44] Abbott B et al 2004 Nucl. Instrum. Methods A 517154

[45] http://www.virgo.infn.it

[46] http://www.geo600.org

[47] Ott C D, Burrows A, Dessart L and Livne E 2006 Phys. Rev. Lett. 96201102

[48] Dimmelmeier H, Ott C D, Marek A and Janka H-T 2008 Phys. Rev. D 78064056

[49] Abdikamalov E B, Ott C D, Rezzolla L, Dessart L, Dimmelmeier H, Marek A and Janka H-T 2009 arXiv:0910.2703

[50] Scheidegger S, Fischer T, Whitehouse S C and Liebendörfer M 2008 Astron. Astrophys. 490231

[51] Marek A, Janka H-T and Müller E 2009 Astron. Astrophys. 496475

[52] Murphy J W, Ott C D and Burrows A 2009 Astrophys. J. 7071173

[53] Kotake K, Iwakami W, Ohnishi N and Yamada S 2009 Astrophys. J. 697 L133

[54] Scheck L, Kifonidis K, Janka H-T and Müller E 2006 Astron. Astrophys. 457963

[55] Van Elewyck V et al 2009 Int. J. Mod. Phys. D 181655

[56] Fritschel P 2003 Gravitational wave detection Proc. SPIE 4856282

[57] Nakamura K 2003 Int. J. Mod. Phys. A 184053

[58] Kistler M D, Yüksel H, Ando S, Beacom J F and Suzuki Y 2008 arXiv:0810.1959

[59] Marrodan Undagoitia T, von Feilitzsch F, Goger-Neff M, Hochmuth K A, Berauer L O, Potzel W and Wurm M 2006 J. Phys. Conf. Ser. 39287

[60] de Bellefon A et al 2006 arXiv:hep-ex/0607026

[61] Autiero D et al 2007 J. Cosmol. Astropart. Phys. 0711011 